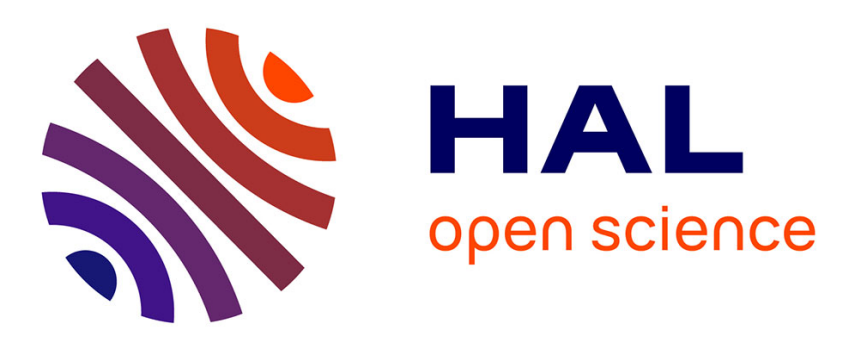

\title{
Les traces des morts : gestion des corps retrouvés et traitement des corps absents à la frontière hispano-marocaine
}

Carolina Kobelinsky

\section{- To cite this version:}

Carolina Kobelinsky. Les traces des morts : gestion des corps retrouvés et traitement des corps absents à la frontière hispano-marocaine. Critique Internationale, 2019, Corps migrants aux frontières méditerranéennes de l'Europe, 83, pp.21-39. 10.3917/crii.083.0021 . hal-03284135

\section{HAL Id: hal-03284135 \\ https://hal.science/hal-03284135}

Submitted on 13 Aug 2021

HAL is a multi-disciplinary open access archive for the deposit and dissemination of scientific research documents, whether they are published or not. The documents may come from teaching and research institutions in France or abroad, or from public or private research centers.
L'archive ouverte pluridisciplinaire HAL, est destinée au dépôt et à la diffusion de documents scientifiques de niveau recherche, publiés ou non, émanant des établissements d'enseignement et de recherche français ou étrangers, des laboratoires publics ou privés. 
Kobelinsky, C. Les traces des morts : gestion des corps retrouvés et traitement des corps absents à la frontière hispano-marocaine, Critique internationale, 2019, 83(2) : 21-39.

Lorsqu'il se remémore sa vie au Maroc dans l'attente de la traversée, Amadou Coulibaly ${ }^{1}$ décrit les conditions précaires des campements, les entraînements quotidiens pour se préparer à escalader les clôtures qui le séparent de l'Europe et la violence de la police à l'égard des personnes migrantes. Il évoque surtout la mort de nombreux compagnons, décédés en tentant de franchir les trois barrières successives qui matérialisent la frontière avec Melilla. Cette ville espagnole enclavée dans la province marocaine de Nador à l'extrémité occidentale de la mer Méditerranée, connue sous le nom de mer d'Alboran, constitue l'une des deux frontières terrestres (l'autre étant l'enclave de Ceuta) entre l'Union européenne et le continent africain. Amadou Coulibaly évoque également les compagnons décédés au Gourougou, petite montagne marocaine qui se trouve à seulement quelques kilomètres de la frontière. Apercevoir l'Europe depuis cette colline lui donnait de l'espoir. Aujourd'hui, il pense encore à ceux qui ne la connaîtront jamais. Au cours des onze mois qu'il a passés dans les campements du Gourougou, il a vu mourir douze personnes. Certaines sont décédées sous ses yeux; les autres, il en a « entendu parler»: «Souleymane est la deuxième personne qu'on a perdue dans la communauté malienne. Il y a eu des morts dans la communauté camerounaise, chez les Guinéens aussi. Il y en a eu beaucoup. (...) Tous les Noirs qui meurent à la barrière, qui meurent au Gourougou, nous on voit pas leurs corps. Rarement on voit les corps. On entend seulement $»^{2}$.

Les corps morts des personnes migrantes décédées aux frontières de l'Europe sont un objet en tension entre plusieurs formes de les considérer et de les traiter. Lorsqu'un corps est retrouvé, l'identité de la personne décédée est tout d'abord une quête. Des habitants des lieux-frontières, des activistes et des personnes migrantes se mobilisent pour recueillir des informations permettant de lui attribuer un nom et contribuer à restituer au défunt une place parmi les siens (sa famille, ses pairs). Il n'existe en effet aucun protocole officiel spécifique visant à redonner un nom et une identité aux corps de celles et ceux qui sont morts aux portes de l'Europe. Qu'il finisse par être identifié ou qu'il demeure sans nom, le corps retrouvé devient une preuve matérielle de la violence du régime contemporain des frontières brandie par les associations qui dénoncent les politiques européennes, une violence sous-estimée ou dépolitisée par certains acteurs étatiques ou intergouvernementaux ${ }^{3}$. Absent, le corps demeure un mystère et le défunt disparu n'existe qu'à travers des apparitions fantomatiques ${ }^{4}$ ou par les témoignages de celles et ceux qui l'ont connu en vie. Invisible, la réalité du corps absent est parfois ignorée, voire contestée. Plusieurs habitantes et habitants de Melilla m'ont ainsi fait part, au cours de

\footnotetext{
1 Afin de préserver l'anonymat et la confidentialité des propos, tous les noms de mes interlocuteurs ont été modifiés.

${ }^{2}$ Tous les entretiens avec Amadou Coulibaly mentionnés ici ont eu lieu à Melilla en novembre 2014.

${ }^{3}$ La question des statistiques en matière de décès aux frontières est à cet égard parlante. L'absence de documentation chiffrée au niveau européen contribue à passer sous silence la réalité de ces décès. Afin de contrer cette forme d'occultation, la société civile et les associations de défense des personnes migrantes a entrepris le comptage pour dénoncer le caractère mortifère des politiques mises en place. Depuis 2013, un organisme intergouvernemental, l'Organisation internationale pour les migrations (OIM), travaille également à la production de statistiques, mais son approche isole les chiffres sur les morts du contexte qui les provoque, dépolitisant complètement la démarche statistique. Voir Charles Heller, Antoine Pécoud, « Compter les morts aux frontières : des contre-statistiques de la société civile à la récupération (inter)gouvernementale », Revue européenne des migrations internationales, 33 (2-3), 2017, p. 63-90.

${ }^{4}$ Le corps absent peut parfois faire irruption, surgir tel un fantôme. Au cours de mon terrain, plusieurs interlocuteurs m'ont raconté des expériences d'apparition de morts aux frontières. Dans de nombreux cas, il s'agissait de morts dont les corps étaient disparus. Ces manifestations fantomatiques de personnes migrantes décédées à la frontière ne seront pas abordées ici.
} 
conversations informelles, de leurs doutes vis-à-vis des personnes qui seraient mortes à la valla (barrière) et dont tout le monde ignorerait le sort.

Le traitement des morts implique bien souvent différents acteurs : policiers, légistes, employés des pompes funèbres et du cimetière. J'observerai ici la façon dont les compagnons de voyage prennent en charge les morts et disparus à la frontière entre Melilla et le Maroc. La mort de Souleymane ${ }^{5}$ permettra de retracer les diverses pratiques mises en place par la « communauté malienne », pour reprendre l'expression d'Amadou Coulibaly, ainsi que ses éventuelles interactions avec les institutions participant à la gestion des défunts. Mon objectif n'est toutefois pas de présenter une étude des spécificités maliennes dans le traitement des défunts au cours de la traversée - cette focale étant le fruit du hasard des rencontres sur le terrain - mais de pointer, à travers ce cas précis, que les morts sont pris en charge par leurs pairs, lesquels, pour beaucoup, organisent leur vie quotidienne aux abords des frontières à partir de leur appartenance nationale. Le matériau ethnographique a été recueilli auprès de personnes migrantes dont la grande majorité sont des hommes ${ }^{6}$, comme auprès de soutiens associatifs et religieux des deux côtés de la frontière au cours de plusieurs séjours entre 2014 et 2018 . Femme au milieu d'interlocuteurs hommes, j'ai rapidement eu accès avec nombre d'entre eux à des discours éloignés des représentations viriles du migrant qui réussit les épreuves du franchissement des frontières. Au cours de nos conversations, leurs récits mettaient plutôt en avant leur vulnérabilité, sans pour autant qu'ils se présentent comme des victimes. La principale langue des échanges était le français, qui n'est pas la langue maternelle de mes interlocuteurs, mais celle dans laquelle les personnes échangeaient entre elles. Cette langue commune est agrémentée de mots d'origines diverses qui nomment des objets, des individus et des actions de leur vie en migration. Un vocabulaire partagé de la frontière se crée ainsi qui emprunte des termes ou des formulations au wolof, au peul, au nouchi (l'argot ivoirien), à l'espagnol, à l'arabe. Je m'appuierai notamment sur une série de conversations informelles et d'entretiens enregistrés que j'ai eus avec Amadou Coulibaly (33 ans) et Gaoussou Dembélé (32 ans), tous deux originaires du Mali. Amadou a quitté son village en décembre 2012. Il est arrivé au Gourougou en novembre 2013 après avoir traversé le nord du Mali, l'Algérie puis le Maroc, où il a d'abord navigué pendant plusieurs semaines entre Fès et Rabat. Gaoussou, lui, a commencé son voyage vers l'Europe un an plus tôt. Il est arrivé au Gourougou en septembre 2013. Tous deux ont réussi à franchir les barrières en octobre 2014. Je les ai rencontrés quelques jours après leur arrivée à Melilla.

Afin d'appréhender à la fois la matérialité du traitement du corps de Souleymane et les récits sur les morts aux frontières qui résident à l'intersection de ce qui est vu ou de ce qu'il est

\footnotetext{
${ }^{5}$ Mes interlocuteurs ont toujours désigné cet homme décédé à la barrière par son prénom (ici modifié).

6 Jusqu'à présent, je n'ai jamais eu la possibilité de construire des liens de confiance avec des femmes ayant fait l'expérience des frontières autour de l'enclave, ni du côté marocain, où je n'ai procédé à des rencontres qu'à partir de connaissances (essentiellement masculines), ni à Melilla, où les femmes restent la plupart du temps dans le CETI (Centro de Estancia Temporal para Inmigrantes), centre de séjour temporaire où les personnes migrantes majeures sont hébergées. Si elles ont le droit d'en sortir, les personnes extérieures n'y ont pas accès. L'absence de légitimité à être dans l'espace public, surtout si elles sont seules et loin de chez elles, explique probablement qu'elles préfèrent rester dans l'enceinte du CETI et n'en sortir que de façon ponctuelle et en groupe. Sur les expériences des femmes en attente de passage de la frontière, voir Elsa Tysler, «"Boza!" disent aussi les femmes », Vacarme, 83, 2018, p. 82-91.

7 Ma recherche sur la prise en charge des morts aux frontières espagnoles de l'Union européenne a démarré en 2013, à partir d'une enquête conduite dans les îles Canaries et les provinces andalouses de Cadix, Alméria et Grenade, où j'ai examiné les trajectoires spatiales des corps retrouvés et les pratiques et motivations des acteurs engagés, de façon officielle ou informelle, dans la gestion de ces morts. Depuis 2014, la majeure partie de mon travail de terrain se déroule autour de l'enclave de Melilla et en suivant certains de mes interlocuteurs dans leurs parcours sur le territoire espagnol. Ce travail est réalisé dans le cadre du programme MECMI, projet ANR-16FRQC-0001.
} 
possible de voir et de ce qui est difficilement perceptible ${ }^{8}$, je mobiliserai la notion de trace. Conçue dans un sens générique comme un fragment d'une entité qui a existé, la trace sera ainsi abordée tout autant comme indice grâce auquel il sera possible d'identifier un corps, de reconstituer une histoire ${ }^{9}$, que comme empreinte voilée, enfuie, qui révèle une absence ${ }^{10}$.

\section{La mort à la barrière et le tracé de la frontière sur le corps}

Souleymane habitait déjà depuis plusieurs mois au Gourougou lorsqu'Amadou Coulibaly et Gaoussou Dembélé y sont arrivés. Ils ne se connaissaient pas avant. Tous les trois étaient originaires de villages voisins, proches de Bamako, au Mali. Le décès de Souleymane a eu lieu un jour de pluie, à l'aube, alors que plusieurs centaines de personnes tentaient de « frapper la barrière », expression utilisée par les personnes migrantes pour désigner le franchissement de la frontière par les clôtures. Assis sur un bout de bois à l'ombre des arbres, Gaoussou Dembélé, une cigarette à la main, évoque ce jour d'un ton maussade, pourtant rare chez cet homme loquace et enjoué, qui décrit volontiers avec humour les épreuves qu'il a traversées : «Il y avait la pluie et quand nous sommes arrivés à la barrière, le trou, comme ils ont fait un trou le long de la barrière maintenant, le trou est profond de quatre mètres et donc ce jour-là il était rempli d'eau parce qu'il pleuvait. Quand nous sommes arrivés nous avons attaqué la barrière. Il n'y avait aucune solution. Certaines personnes se sont jetées dans l'eau afin de se débrouiller et sortir et escalader la barrière. C'est ce jour-là que Souleymane est mort. Parce que ce trou, dans certains coins ils ont mis du barbelé et ils l'ont couvert de sable. Et comme le trou est rempli d'eau [à cause de la pluie], ce barbelé peut à certains endroits sortir du sable. Le gens se sont jetés dans le trou en espérant sortir et comme ça, Souleymane, paix à son âme, est tombé dans l'eau. Il est décédé dans l'eau comme ça ${ }^{11}$.

Amadou Coulibaly, qui profite lui aussi de l'ombre des arbres et acquiesce de la tête à chaque phrase de son compatriote, prend alors la parole : «Je l'ai vu, moi, d'un coin de l'œil pendant que je courais, Souleymane, je l'ai aperçu tomber dans l'eau [silence] dans ce trou qu'ils ont fait pour nous créer un obstacle supplémentaire, pour nous arrêter. Lui, ils l'ont arrêté ${ }^{12}$

Le « trou » dont parlent Gaoussou et Amadou est un fossé creusé à certains endroits stratégiques entre la première barrière et la route du côté marocain de la frontière. Il complète un dispositif qui ne cesse de se renforcer depuis 1998, date du début de la construction d'une barrière de presque $12 \mathrm{~km}$, couvrant la totalité de la frontière terrestre. Cette construction a été un tournant dans le processus de «frontiérisation ${ }^{13}$ de Melilla, dans sa transformation en frontière européenne ${ }^{14}$. Une deuxième barrière de trois mètres de haut a été ensuite érigée parallèlement à la première. En 2005, en réponse à plusieurs tentatives de passage au cours desquelles des

\footnotetext{
${ }^{8}$ Valentina Napolitano, « Anthropology and Traces », Anthropological Theory, 15 (1), 2015, p. 47.

${ }^{9}$ Dans l'esprit du paradigme indiciaire de l'historien Carlo Ginzburg. Voir Carlo Ginzburg, « Signes, traces, pistes. Racines d'un paradigme de l'indice », Le Débat, VI (6), 1980, p. 3-44 ; C. Ginzburg, Mythes, emblèmes, traces. Morphologie et histoire, Paris, Flammarion, 1986.

${ }^{10}$ Elisabeth Anstett, Luba Jurgenson, « Introduction - Héritages et mémoires du Goulag : pour une anthropologie de la trace », dans E. Anstett, L. Jurgenson (dir.), Le Goulag en héritage : pour une anthropologie de la trace, Paris, Petra, 2009.

${ }^{11}$ Tous les entretiens avec Gaoussou Dembélé mentionnés ici ont eu lieu à Melilla en novembre 2014.

${ }^{12}$ Entretien avec Gaoussou Dembélé.

${ }^{13}$ Paolo Cuttitta, «La "frontiérisation” de Lampedusa, comment se construit une frontière », L'Espace politique, 25 (1), 2015 (en ligne).

${ }^{14} \mathrm{La}$ 《frontiérisation » de Melilla a cependant commencé plus tôt, au moment de l'entrée de l'Espagne dans l'Union européenne (1986) et de la promulgation un an plus tôt de la loi en matière d'immigration en 1985, qui induisait - non sans réticences - un processus de régularisation administrative et de naturalisation pour les personnes d'origine marocaine qui habitaient depuis des décennies dans les enclaves de Melilla et de Ceuta, mais n'avaient pas de citoyenneté ni de résidence espagnole.
} 
balles tirées de part et d'autre de la frontière ont blessé des centaines de personnes et tué au moins onze migrants ${ }^{15}$ - période qualifiée par les médias espagnols de "crisis de las vallas [crise des barrières] »-, le gouvernement a décidé de rehausser les grillages à six mètres et de construire un cordage tridimensionnel, enchevêtrement de câbles connu sous le nom de troisième barrière et dont le but est d'empêcher le passage entre la première et la deuxième barrière $^{16}$. À ces clôtures s'ajoutent des barbelés qui les longent ainsi qu'un réseau de câbles souterrains relié à des capteurs électroniques de bruit et de mouvement. La surveillance est complétée par un système de caméras vidéo de vision nocturne. Des postes de surveillance sont répartis le long des grillages et les routes entre les barrières permettent le passage constant de véhicules de la Guardia Civil ${ }^{17}$ qui patrouillent la frontière. Du côté marocain, celui des barrières, des agents de la Gendarmerie royale effectuent également des rondes ${ }^{18}$. En 2014 , quelques mois avant la tentative au cours de laquelle Souleymane a trouvé la mort, le Maroc a entrepris de creuser le fossé dont parlent Gaoussou Dembélé et Amadou Coulibaly ${ }^{19}$.

« Frapper la barrière » est une entreprise collective organisée par le chef d'une communauté qui s'appuie sur l'avis de plusieurs conseillers. Les campements du Gourougou sont organisés par communauté nationale. Chacune a son emplacement, et chacune est dirigée par un chef : "Chez les Maliens, l'élection du chef dépend de la sagesse et de la générosité et la solidarité de la personne. Souvent ça dépend aussi de l'ancienneté, combien de temps tu as fait dans la forêt. Et ce n'est pas seulement ça, parce qu'il faut aussi être gentil, être légal, être fort dans le mental $»^{20}$. Le jour où Souleymane est décédé, la tentative était menée par les Maliens. Selon la règle, la communauté avait appris la décision de tenter le franchissement de la frontière au dernier moment afin d'éviter que l'information ne fuite : «Si la police l'apprend, ils vont nous attendre tous prêts, il n'y aura plus de surprise. Notre force c'est de les surprendre $»^{21}$. Comme à chaque fois que la frontière est «frappée », ils sont descendus de la colline dans la nuit, de façon ordonnée, en file. Ils ont couru vers la frontière, sans se parler, en faisant le moins de bruit possible : «On entend les bruits de la nuit, les feuilles qui craquent sous les pieds et les battements du cœur qui vont vite vite $\gg^{22}$. À quelques mètres de la première barrière, ils se sont souhaité bonne chance et se sont cachés. Comme convenu, c'était l'appel à la prière de l'aube émis par les mosquées voisines qui indiquerait qu'il était temps de démarrer la course : «On court parmi les rochers, dans les fossés, tout ça. Beaucoup se cassent [terme de Gaoussou] les pieds en courant dans le noir, c'est un grand effort, il y en a qui tombent malades. Et si t'as pas un frère, un frère digne de ce nom, tout le monde va t'abandonner dans la forêt. Tu peux peut-

\footnotetext{
${ }^{15}$ Migreurop, Guerre aux migrants. Le Livre noir de Ceuta et Melilla, Migreurop, 2006.

16 Parallèlement, le Plan África se met en place à partir de 2006 pour répondre cette fois à ce que les médias espagnols nomment la «crisis de los cayucos [crise des pirogues]». Cette disposition généralise la signature d'accords de réadmission de migrants et de coopération dans le contrôle des frontières des pays tiers africains d'où partent les bateaux, entre autres, le Sénégal et la Mauritanie. Asociación Pro Derechos Humanos de Andalucía (APDHA), Derechos humanos en la frontera sur 2007, Séville, APDHA, 2007, et Derechos humanos en la frontera sur 2014, Séville, APDHA, 2014.

${ }^{17}$ Force de police à statut militaire, comparable à certains égards à la gendarmerie nationale française, la Guardia Civil exerce des missions multiples : sécurité publique, police judiciaire, missions à caractère militaire et relevant du domaine fiscal.

18 À la dimension répressive de ce dispositif technologique s'ajoute celle concernant les bénéfices économiques puisque le contrôle et la surveillance des frontières sont de véritables «industries », voire des «business », profitables. Ruben Andersson, Illegality Inc. Clandestine Migration and the Business of Bordering Europe, Berkeley, University of California Press, 2014 ; Claire Rodier, Xénophobie business. À quoi servent les contrôles migratoires ?, Paris, La Découverte, 2012.

${ }^{19}$ APDHA, Derechos humanos en la frontera sur 2016, Séville, APDHA, 2016, p. 53.

${ }^{20}$ Entretien avec Gaoussou Dembélé.

${ }^{21}$ Conversation avec Adama Touré (29 ans), ivoirien, qui a participé à plus d'une dizaine de tentatives, Melilla, le 4 novembre 2014.

${ }^{22}$ Conversation avec Modibo Diabaté (22 ans), malien, qui a également fait l'expérience de nombreuses tentatives, Melilla, le 28 mai 2016.
} 
être mourir. Mais si tu réussis à avancer, quand on arrive près de la barrière, on se cache et on choisit le bon moment pour aller la frapper. Avec la prière, quand on l'entend, on donne le cri "yallah", fonçons [terme de Gaoussou]. Si on voit la police, on crie encore plus fort. Et les forces marocaines, même s'ils sont des millions, ils ne pourront pas nous stopper parce que nous sommes déterminés et, surtout, parce que nous sommes prêts à mourir. Oui, on peut mourir et on est prêts à risquer notre vie, vraiment $»^{23}$.

À partir de ce moment, pour certains, c'est une question de chance, pour d'autres, c'est la volonté de Dieu. Pour tous, c'est surtout une question de rapidité. Les expériences répétées et celles des autres bien avant ont permis d'affiner la technique et de chronométrer la course. Plusieurs interlocuteurs m'ont dit qu'il fallait atteindre la première barrière en moins de sept minutes. Passé ce délai, impossible d'échapper aux gendarmes marocains. Ensuite, ils ont trois minutes pour franchir les trois clôtures et atteindre le territoire espagnol. Passé ce délai, ce sont les agents de la Guardia Civil qui cette fois les arrêteront immanquablement ${ }^{24}$. La confrontation avec les forces de l'ordre devient ainsi presque inexorable : « Nous sommes obligés d'affronter les militaires marocains, nous sommes forcés à de les affronter. Et nous les affrontons à main nue, haut la main ${ }_{2}$ on les affronte pacifiquement, sans violence. Ce sont eux qui nous affrontent violemment. Dès qu'ils nous voient venir ils prennent des cailloux, ils n'ont pas de matraques, mais des bois, des fers et ils nous lapident avec ça. Et donc nous on n'a pas le choix, il faut avancer, on est face au mur, on doit avancer. Ils nous poussent, nous lancent des cailloux. Mais comme nous sommes déterminés, on arrive à les disperser et à nous faire une ouverture. Ceux qui arrivent à se glisser dans les ouvertures peuvent commencer à escalader. Ceux qui n'ont pas la chance, eux ils restent en bas. C'est la catastrophe maintenant qui commence. Ils vont te taper. Ils te tapent partout, ils ne choisissent pas où taper, ils le font partout. S'ils te tapent sur la tête, ils peuvent même te briser la tête. Donc en essayant de protéger notre tête on se casse la main, le bras. En essayant de se courber encore on se casse les pieds, les jambes. À force de taper sur certaines zones, ils peuvent aller jusqu'à la mort. Tu vois les frères par terre lancer toutes sortes de cris. Et pendant ce temps-là, ceux qui ont réussi à grimper les six mètres de hauteur peuvent parfois tomber. Si t'as pas la chance tu peux te casser le cou, si t'as pas la chance tu vas te briser la colonne vertébrale. On a vu toutes sortes de morts, il y en a qui tombent dans le trou d'une profondeur de 4 mètres. Et il y a des barbelés partout, mais on fonce, on fonce sur les barbelés, on fonce sur les forces marocaines. Les barbelés nous déchirent la peau. Tout ça nous fait des cicatrices. Il y a beaucoup de morts qui ont été enregistrés là. Beaucoup de Noirs sont tués comme ça, simplement parce qu'ils veulent quitter le Maroc et entrer de l'autre côté, en Europe. Ça dépasse l'imagination $»^{25}$.

L'expérience de la frontière implique, pour les personnes migrantes, une « connaissance par corps $\gg^{26}$ qui garantit une compréhension pratique des situations fréquemment vécues autour de l'enclave. Tous ceux qui arrivent au Gourougou apprennent très vite que, pour mettre les chances de leur côté et réussir le franchissement des barrières, il faut entraîner son corps, l'endurcir pour résister à cette confrontation corps à corps avec les forces de l'ordre, l'exercer pour courir et escalader en vitesse : « Nous sommes des soldats aguerris ${ }^{27}$. Au Gourougou, il y

\footnotetext{
${ }^{23}$ Entretien avec Gaoussou Dembélé.

${ }^{24}$ Une fois sur le sol espagnol, il faut courir pour s'éloigner des clôtures afin d'éviter d'être expulsé de façon immédiate vers le Maroc. Connue sous le nom de «devolución en caliente » (littéralement, retour à chaud), cette pratique est largement critiquée pour être contraire à la Convention européenne des droits de l'homme. Couramment employée dans les enclaves de Melilla et Ceuta depuis plusieurs années, elle a été légalisée en 2015 avec l'introduction de la notion du « rechazo en frontera » (expulsion à la frontière).

${ }^{25}$ Entretien avec Gaoussou Dembélé.

${ }^{26}$ Pierre Bourdieu, Méditations pascaliennes, Paris, Le Seuil, 2003 (1997), p. 185-234.

${ }^{27}$ Plusieurs interlocuteurs expriment leurs expériences de la frontière à travers des métaphores guerrières. Je ne m'y attarderai pas ici, mais il me semble que, au-delà de la dimension virile qu'elles comportent, ces images renvoient à l'intériorisation de la dimension guerrière des politiques migratoires européennes et à la façon dont
} 
a tout : « Nous montons des collines chaque jour, nous portons de bidons d'eau, des fagots, du poids sur la tête $»^{28}$. Un jeune Guinéen m'a dit en rigolant et en montrant les agents marocains que l'on devinait au loin, de l'autre côté des barrières : « Nous sommes bien formés, bien plus que cette force-là ! ». À ce « dressage du corps ${ }^{29}$ s'ajoute la préparation des objets nécessaires à l'escalade des clôtures. Il faut d'abord se procurer des vis qui seront incrustées dans les semelles des chaussures pour en faire des crampons permettant ainsi de prendre appui sur les clôtures. Ensuite, il faut trouver des bouts de fer qui seront recourbés pour en faire des crochets qui permettront de mieux s'agripper au cours de l'ascension. Une fois les objets préparés, «tu te concentres sur le moral, le tien, celui des autres, il faut se remonter le moral, se dire qu'on va y arriver, que ce sera boza $»^{30}$.

Ce jour-là, Souleymane était prêt pour «frapper la barrière », ses chaussures étaient plantées de vis, des crochets étaient attachés à ses manches et, selon l'expression d'Amadou Coulibaly, « il avait l'envie ». Cela n'a pas suffi.

De retour au Gourougou, peut-on tracer les disparus?

Après des heures d'affrontements avec la gendarmerie marocaine et la Guardia Civil, la frontière retrouve son calme apparent, aucun indice ne permet d'imaginer ce qui vient de se passer. On ne voit que « le nombre habituel de véhicules des forces de l'ordre circulant sur les routes ici ou là-bas », me dit un activiste habitant Melilla tandis que nous évoquons longuement cette tentative $^{31}$. Gaoussou Dembélé, lui, se souvient que l'opération n'a pas duré très longtemps cette fois-là, en comparaison avec ce qu'il avait déjà connu. Beaucoup de personnes ont été arrêtées avant même d'atteindre la première barrière. Lorsqu'ils sont de retour au Gourougou, affaiblis et bouleversés, ceux qui n'ont pas réussi à gagner le territoire espagnol s'occupent de soigner les blessés et de faire le bilan des absents. S'ils ne sont pas dans le campement, où sont-ils ? Lorsqu'il y a eu boza, la nouvelle se répand à toute allure. On sait très vite qui est arrivé à Melilla. Ce jour-là, personne n'a réussi à passer de l'autre côté. Pour ceux dont on n'a aucune nouvelle, il faut activer les réseaux de connaissances. Il faut essayer de retrouver leur trace. Ils ont peut-être été conduits de force par les autorités marocaines dans des villes loin du Gourougou. Presque tous mes interlocuteurs ont fait l'expérience de déplacements contraints vers Fès, Agadir, Rabat ou encore vers la frontière algérienne, après une tentative de passage des clôtures ${ }^{32}:$ «Mais ce jour-là nous avons contacté tous, tous les amis : "Est-ce qu'ils ont vu Souleymane?". Toutes les personnes que nous avons contactées nous disent: "Non. Non. Non. Non, nous n'avons pas vu Souleymane" $»^{33}$.

Souleymane a disparu. Gaoussou, Amadou et leurs compagnons d'infortune se disent qu'il est peut-être mort. Il devient un disparu de plus et son histoire rejoint toutes celles qui circulent depuis des années des deux côtés de la frontière sur les morts de la barrière. Racontés non seulement par des personnes migrantes mais aussi par des membres d'associations de soutien

celle-ci les conduit à réagir, à devenir des «soldats ». Pour une analyse des politiques migratoires en termes de « guerre aux migrants », voir Marc Bernardot, Captures, Bellecombe-en-Bauges, Éditions du Croquant, 2012.

${ }^{28}$ Entretien avec Amadou Coulibaly.

${ }^{29}$ Marcel Mauss, «Les techniques du corps », dans M. Mauss, Sociologie et anthropologie, Paris, PUF, 1993, p. 365-386.

${ }^{30}$ Entretien avec Gaoussou Dembélé. On entend souvent les migrants crier « boza ! boza !» lorsqu’ils essaient de sauter les clôtures. Selon certains interlocuteurs, cela voudrait dire « victoire » et viendrait d'une déformation d'un terme wolof.

${ }^{31}$ Conversation avec Enrique (53 ans), Melilla, le 4 avril 2016.

${ }^{32} \mathrm{C}$ 'est aussi ce qui ressort des témoignages recueillis quelques années plus tôt dans le rapport Migreurop, Guerre aux migrants. Le Livre noir de Ceuta et Melilla, op. cit.

${ }^{33}$ Entretien avec Gaoussou Dembélé. 
et des salariés des institutions de prise en charge à Melilla, ces récits parlent de ceux qui meurent à chaque tentative de passage et dont les corps ne sont jamais retrouvés. Toutes les histoires que j'ai entendues disent la violence de cette traversée, de l'affrontement avec les forces de l'ordre. Les nombreuses hypothèses émises sur la disparition - les corps seraient mangés par des animaux, phagocytés par la terre, enfouis dans des fosses communes - reflètent l'incertitude qui entoure le sort des dépouilles. Elles révèlent également combien la disparition fait partie de l'imaginaire collectif de la frontière en tant qu'angoisse ultime qui viendrait enfoncer irrémédiablement les personnes migrantes dans une invisibilité dont elles font déjà en partie l'expérience. Invisibilité dont elles cherchent pourtant à s'extraire par la migration ${ }^{34}$.

$S$ 'ils comportent une forte dimension anxiogène, ces récits permettent de faire exister ceux qui ne sont plus là : «C'est peut-être pour ne pas oublier les morts », s'interroge à voix haute Cellou Diallo, Peul originaire de Guinée, au sujet des histoires qu'il a entendues depuis son arrivée au Maroc et qu'il transmet à son tour à qui veut bien les recueillir ${ }^{35}$. La narration devient ici une trace doublement temporelle en ce qu'elle est à la fois témoignage des vies (et des morts) passées et hantise immuable concernant une possibilité future ${ }^{36}$. Comme toutes les personnes qui racontent ces disparitions à la barrière, Cellou Diallo se fait le porte-parole des absents et à travers eux d'une histoire collective, celle de la traversée des frontières et de ses épreuves létales.

Le seul qui « manque à l'appel » après cette tentative est, selon les mots d'Amadou Coulibaly, «dans la tête de tous », lorsque la nuit tombe et qu'ils s'apprêtent à dormir dans leurs « bunkers », ces tentes qu'ils ont construites avec des tronçons d'arbres protégés par de grandes bâches en plastique achetées dans les boutiques de Beni Enzar ou de Nador, les villes voisines. Le lendemain, Amadou descend avec quelques autres compagnons à Beni Enzar pour chercher de quoi manger dans les poubelles, éventuellement faire la manche, ce que la plupart de mes interlocuteurs appellent «taper Salam ». L'un d'eux contacte à nouveau un groupe de Maliens qui se trouvent à Fès au cas où ils auraient des nouvelles de Souleymane. Toujours rien. La nuit tombe à nouveau, et Gaoussou Dembélé s'allonge par terre avec ses deux couvertures en pensant qu'il n'y a plus d'espoir, que Souleymane est mort. Comme chaque nuit, des « veilleurs » vont rester debout pour s'assurer que tout est calme sur le Gourougou et que les hommes qui se trouvent dans les campements sont en sécurité ${ }^{37}$. La police marocaine mène fréquemment des opérations d'évacuation afin d'éparpiller et de désorganiser les communautés. Et ces opérations peuvent se révéler tragiques. Deux semaines avant la disparition de Souleymane, lors d'une évacuation violente, un autre compagnon est décédé : «Les forces marocaines sont venues au Gourougou à deux heures du matin, on a coupé notre sommeil, on dormait. On a commencé à prendre nos caoutchoucs, nos marmites, nos affaires, on a commencé à courir pour monter dans la montagne, ils sont venus nous chasser, on courait, ils couraient derrière. De deux heures du matin jusqu'à six heures, ils nous encerclaient. On court, on court, on court. En courant il y avait un petit, Idrissa, qui a été arrêté parce qu'il vient d'arriver, c'est un nouveau, Malien. On était ensemble en même temps, on était caché et on a commencé à courir. Il a dit : "Ils sont beaucoup". Et a reculé. Ils lui ont dit de s'arrêter, mais le petit, il n'a pas voulu s'arrêter. Quand il s'est arrêté, un homme de la force marocaine est venu

\footnotetext{
${ }^{34}$ Sur la forme et le contenu de ces histoires sur les morts sans corps, voir Carolina Kobelinsky, « Exister au risque de disparaître. Récits sur la mort pendant la traversée », Revue européenne des migrations internationales, 33 (23), 2017, p. 115-131.

${ }^{35}$ Conversation avec Cellou Diallo (18 ans), Melilla, le 24 mai 2016.

${ }^{36}$ Sarah Green, «Lines, Traces and Tidemarks: Reflections on Forms of Borderli-ness », EastBordNet, COST Action IS0803 Working Paper, Helsinki, 2009.

37 À l'époque, il n'y avait pas de femmes dans le campement malien. Au cours des treize mois qu'il a passés au Gourougou, Gaoussou Dembélé se souvient pourtant qu' « il y avait 25 femmes au Gourougou à un moment donné, deux étaient enceintes et deux autres avaient des bébés. Dans le campement du Nigeria, il y avait quatre femmes, une avec un bébé, un petit d'un an et demi plus ou moins ». Entretien avec Gaoussou Dembélé.
} 
le pousser. Le petit est tombé directement, nos frères noirs qui étaient à côté ont vu ça aussi $\gg^{38}$. Lorsque les forces de l'ordre ont quitté les lieux et que le Gourougou a retrouvé une tranquillité apparente, ceux qui avaient vu la scène ont crié le nom du jeune, mais n'ont pas obtenu de réponse. Peu à peu, les personnes qui avaient réussi à s'échapper, celles qui s'étaient cachées, sont revenues mettre en ordre ce qui restait des campements ${ }^{39}$. Elles ont vérifié que les quelques objets qu'elles avaient dissimulés dans les arbres, sous des cailloux, étaient toujours là, si les sacs de riz et les boîtes de sardines n'avaient pas été pillés. D'après les nombreux récits recueillis, saccager les affaires des habitants des campements est un des objectifs de ces opérations policières. Le lendemain, lorsque quelques personnes sont descendues vers la pompe à pied du mont où il est possible de s'approvisionner en eau, un « Marocain qui a des moutons » les attendait. Il leur a dit qu'il avait vu un cadavre et qu'il s'agissait d'un «camarade». Quelqu'un a passé alors un coup de fil pour donner la nouvelle et la faire circuler. Peu de temps après, Amadou et deux autres Maliens, dont le chef de la communauté, sont descendus et ont été guidés par le berger vers le corps : «Les Arabes qui étaient là, ils nous ont aidé à appeler [la police] et à appeler les sapeurs-pompiers. On a pris nos téléphones et on a filmé, après on a caché nos téléphones. Ils sont venus, ils nous ont amené au commissariat, ils ont tapé le PV pour nous, ils nous ont demandé comment il était mort, nous on a dit : "Les bomboula [forces de l'ordre $]^{40}$ sont venus à quatre heures du matin, on a couru dans la montagne, on est restés làbas, et puis lui on l'a pas vu, un jour, deux jours, on s'est dit que quelque chose lui était arrivé". On a dit ça, nous, et ils nous ont dit de partir. Si on disait que c'était eux, que c'était les forces marocaines, ils allaient nous tuer, mais on a été intelligents. Mais nous on sait, on sait que c'est eux qui l'ont tué. Dieu aussi sait. Et eux aussi le savent mais nous, on ne peut pas déclarer. Parce que si on déclare, c'est nous qui devenons un cadavre ${ }^{41}$

Que ces hommes déposent une plainte auprès des forces de l'ordre qui, la nuit précédente, ont tenté de les chasser peut surprendre. Dénoncer la mort d'un pair auprès des autorités répond à la volonté d'inscrire officiellement une trace du défunt, de faire reconnaître sa réalité et de lui accorder - ne serait-ce qu'à travers une démarche administrative - une forme d'existence, malgré le silence qui protège ceux qui sont responsables de sa mort.

Le décès du «petit » Idrissa était le premier dans la communauté malienne depuis l'arrivée d'Amadou Coulibaly au Gourougou. Sa mort était «dans toutes les mémoires » lorsque Souleymane a disparu.

\section{Les traces des corps, enjeu d'identification}

Trois jours après la disparition de Souleymane, un jeune homme d'un campement du Gourougou a reçu un appel sur son portable. C'était un prêtre du diocèse de Tanger à Nador, avec lequel le jeune était en contact depuis plusieurs mois, qui souhaitait informer la communauté que, comme l'explique Gaoussou Dembélé, « les militaires marocains avaient vu le corps d'un jeune homme qui était mort dans l'eau durant la tentative quelques jours auparavant et que le corps se trouvait à Nador».

Ce prêtre jésuite originaire des îles Canaries était à l'époque le responsable de la Délégation des migrations du diocèse de Tanger à Nador, institution créée en 2011 afin de proposer une aide et un accompagnement minimal aux personnes migrantes qui se trouvaient dans la région dans l'attente de réussir la traversée vers l'enclave espagnole. Faisant le bilan des corps

\footnotetext{
${ }^{38}$ Entretien avec Amadou Coulibaly.

${ }^{39}$ Lors de ces descentes au Gourougou, des migrants peuvent être éloignés de force de la région de Nador.

${ }^{40}$ Dans ce contexte, bomboula désigne les forces de l'ordre, mais le terme (ainsi que ses variantes boumbla ou bombola) est également utilisé pour nommer les opérations de ratissage et d'évacuation des campements conduites par les forces de l'ordre.

${ }^{41}$ Entretien avec Amadou Coulibaly.
} 
retrouvés au cours des trois années d'activités de la Délégation à la frontière avant d'être interdit de séjour au Maroc, il se souvient de ce coup de fil : « On reçoit un appel [à la Délégation] pour nous dire que le corps d'un Subsaharien se trouve à la morgue de l'hôpital [El] Hassani. Les légistes ne pouvaient pas l'identifier. J'appelle alors un jeune qui est au Gourougou pour le lui dire. Très vite, deux heures plus tard, il me rappelle, me dit que les Maliens n'ont pas de nouvelles d'un des leurs, que deux personnes vont descendre pour se rendre à la morgue. Nous les accompagnons $\gg{ }^{42}$.

Aucune trace, aucun indice sur le corps ne permet l'identification médico-légale du cadavre de cet homme. Le prélèvement d'empreintes digitales n'apporte pas d'information puisque le défunt - comme toutes les personnes migrantes en route pour l'Europe, à quelques exceptions près - n'a pas été enregistré au Maroc ni sur aucune base de données européenne. Les prélèvements $\mathrm{ADN}$ ne sont pas courants et n'apporteraient de toute façon pas grand-chose à ce stade de l'enquête puisqu'il n'y pas d'échantillon ante mortem du défunt ni d'un membre de la famille permettant de procéder à une comparaison. Les médecins ne peuvent alors qu'attribuer un âge approximatif à ce corps pour eux insondable. Ce sont les deux délégués envoyés par la communauté qui permettent son identification. Je ne sais pas exactement comment cela s'est passé, mais les récits que j'ai entendus rapportaient que la reconnaissance n'avait pas été difficile. Ils ont très vite affirmé qu'il s'agissait du corps de Souleymane. Pour ce qui est du «petit» Idrissa, c'est une cicatrice au cou qui, telle une trace indiciaire, a permis à Amadou Coulibaly de confirmer que le corps très dégradé qui avait été retrouvé en bas de la colline était le sien. Après avoir signé, auprès de la police, la déclaration reconnaissant le défunt qui leur avait été présenté, les deux délégués de la communauté malienne sont retournés dans le campement pour annoncer la nouvelle. Souleymane n'était plus un disparu. Il était mort et son corps se trouvait pour l'instant à la morgue de Nador.

Si Souleymane a pu être identifié, c'est grâce à une chaîne de collaboration mise en place grâce à une série d'appels téléphoniques : un agent de la police appelle la Délégation des migrations, le prêtre de la Délégation appelle quelqu'un au Gourougou. D'après les récits de plusieurs interlocuteurs, il arrive que des corps soient retrouvés du côté marocain de la barrière mais que les autorités ne contactent aucune institution ou association travaillant auprès de personnes migrantes, ce qui accroît l'incertitude concernant les disparus. L'identité et le sort des dépouilles deviennent alors plus difficiles à retracer. Aux frontières espagnoles, c'est également par des moyens artisanaux et peu élaborés que l'identification de migrants décédés peut avoir lieu. Des activistes se mobilisent afin de contribuer aux efforts d'identification en faisant circuler les informations concernant un décès qu'ils obtiennent des légistes, de la police ou de la Croix-Rouge. Les personnes migrantes rencontrées des deux côtés de la frontière m'ont toutes fait part de leur mobilisation afin de contrer la mort anonyme, notamment à travers Facebook, WhatsApp et les appels téléphoniques. Les smartphones et plus largement les technologies de la télécommunication touchent tous les aspects de la vie et de la mort des personnes migrantes en route pour l'Europe ${ }^{43}$. Ils permettent non seulement de se tenir informé des routes et des opportunités au cours du parcours ou en Europe, mais aussi de retrouver ou de garder la trace des morts, comme lorsqu'Amadou Coulibaly filme le corps inerte du «petit » Idrissa.

Les formes contemporaines de surveillance et les nouvelles technologies de contrôle aux frontières peuvent augmenter ou au contraire diminuer l'anonymat de celles et ceux qui les traversent. Les hommes et les femmes qui essayent d'arriver en Europe sans les autorisations nécessaires font l'expérience de toutes sortes de procédés visant à les identifier. Fichés, repérables, ils sont gouvernés par la trace ${ }^{44}$. Celle-ci devient à la fois un outil et un objectif des

\footnotetext{
${ }^{42}$ Entretien avec l'ancien responsable de la Délégation des migrations à Nador, Málaga, le 26 avril 2016.

${ }^{43}$ Dana Diminescu, « Traces numériques », Plein droit, 110 (3), 2016, p. 3-6.

${ }^{44}$ Armand Mattelart, « Gouverner par la trace », Mouvements, 62 (2), 2010, p. 11-21.
} 
politiques. Elle permet in fine de les expulser. La trace des morts peut au contraire disparaître à jamais. Non pas en raison d'une absence d'indices - les empreintes digitales résistent souvent à la mort -, mais parce qu'ils ne sont pas tracés. La traçabilité ${ }^{45}$ des défunts n'est pas un objectif des politiques. À la multiplicité de technologies de plus en plus sophistiquées visant l'identification des vivants - dans le but de les trier, de les confiner et finalement de les contrôler - s'oppose l'absence de système commun au niveau européen pour faciliter l'identification des personnes migrantes décédées « au passage ». À la traçabilité des vivants s'oppose l'anonymat des morts. Aucun système spécifique n'existe non plus au niveau national, dans aucun des pays de l'Union européenne ni dans les pays voisins. Les enquêtes officielles visant à l'identification des corps retrouvés sont rares ou partielles puisqu'elles n'ont lieu que si elles font partie d'une investigation criminelle plus large dont l'objectif principal est de poursuivre les passeurs, considérés comme étant les seuls responsables des décès ${ }^{46}$. Les corps retrouvés deviennent l'indice de l'opposition entre différents acteurs : celles et ceux qui, au niveau local, tentent de contribuer à leur identification et les concepteurs des politiques aux niveaux national et européen, qui sont bien souvent indifférents au sort des morts aux frontières et contribuent ainsi à une forme de «nécro-violence ${ }^{47}$. Les corps morts révèlent « la façon dont l'importance spécifique de la [des] personne[s] décédée[s] est (diversement) construite » par les acteurs impliqués dans leur traitement ${ }^{48}$.

De la morgue de l'hôpital El Hassani à Nador, le corps de Souleymane est transporté vers un cimetière juste en dehors de la ville où il est enterré en présence de quelques membres de la Délégation des migrations ${ }^{49}$. Le prêtre ramasse par terre deux ou trois petits cailloux qu'il gardera en hommage au défunt, comme il le fait à chaque fois qu'il accompagne le dernier voyage de personnes migrantes décédées à la frontière. Le tas de cailloux que conserve le prêtre devient lui aussi une trace, une représentation de ces vies perdues à la frontière. Amadou Coulibaly et Gaoussou Dembélé ne se sont jamais rendus devant sa tombe. Peut-être à cause de la peur de se faire arrêter et embarquer par la police de la ville de Nador. Peut-être parce qu'ils ont préféré lui témoigner leur considération ailleurs, autrement. L'itinéraire post mortem du corps du «petit» Idrissa demeure quant à lui incertain. Les membres de la communauté malienne n'ont pas été informés du devenir de la dépouille dont ils ont seulement appris qu'elle avait été conduite à la morgue de Nador.

\section{Honorer les morts, garder leur trace}

\footnotetext{
45 Traçabilité entendue à la manière du terme anglais traceability, comme l'aptitude au traçage.

${ }^{46}$ Virginie Guiraudon, «Lutte contre les passeurs : une politique qui ne protège pas les victimes de l'immigration illégale », dans Claire Rodier, Emmanuel Terray (dir.), Immigration : fantasmes et réalité. Pour une alternative à la fermeture des frontières, Paris, La Découverte, 2008. Babels, «Introduction », dans Babels, La mort aux frontières de l'Europe : retrouver, identifier, commémorer, coordonné par Carolina Kobelinsky et Stefan Le Courant, Neuvy-en-Champagne, Le passager clandestin, 2017, p. 7-36.

${ }^{47}$ Par ce terme, Jason De León désigne la violence performée et produite à travers le mauvais (ou le non-) traitement des cadavres des personnes migrantes décédées en tentant de traverser le désert qui sépare le Mexique des États-Unis. Jason De León, The Land of Open Graves. Living and Dying on the Migrant Trail, Berkeley, University of California Press, 2015.

${ }^{48}$ Katherine Verdery, The Political Lives of Dead Bodies. Reburial and Postsocialist Change, Chichester, Columbia University Press, 1999, p. 28.

${ }^{49}$ Les personnes migrantes décédées dont on sait qu'elles étaient de confession chrétienne sont parfois enterrées, d'après ce que m'a expliqué l'ancien responsable de la Délégation des migrations, dans l'un des cimetières chrétiens gérés par le consulat espagnol en territoire marocain. De l'autre côté de la frontière, en Espagne, c'est dans des cases de columbarium dans les murs des cimetières municipaux des lieux-frontières que sont inhumés la plupart des migrantes et des migrants décédés. Les fossoyeurs inscrivent parfois le mot « inmigrante » sur le ciment frais, suivi de la date. Parfois, on retrouve une inscription indiquant la nationalité du défunt. Il est rare qu'une plaque indique son nom. À Melilla, certains sont enterrés dans le cimetière musulman qui se trouve juste à la frontière.
} 
Le jour où a été confirmé le décès de Souleymane reste gravé dans la mémoire d'Amadou Coulibaly et de Gaoussou Dembélé : «C'était un jour très triste, on venait de perdre un élément, un ami avec qui on discutait tous les jours, avec qui on faisait du thé tous les jours avec qui on bavardait tous les jours. C'était très triste. Ça nous a beaucoup touché le cœur ${ }^{50}$.

Si la mort fait partie de la vie quotidienne au cours de la traversée - qu'elle apparaisse comme une éventualité ou qu'elle s'impose avec toutes les personnes que l'on a vues périr dans le désert, en mer ou à la barrière -, le moment où l'on apprend le décès d'un pair condense des émotions multiples. La douleur devant cette perte s'accompagne de l'angoisse de se savoir fragile, d'une exacerbation de la crainte de mourir pendant le franchissement des frontières : « Ce jour nous avons tous eu au Gourougou très très peur de la barrière. Tu sais que tu peux mourir, qu'il y a des frères qui meurent. Là c'était trop proche. Personnellement je me disais : "Si aujourd'hui c'est Souleymane peut-être que demain ce sera moi. Ou peut-être que demain ce sera l'autre ou l'autre ou l'autre". Le fait de vivre au Gourougou, j'avais tellement peur parce que je ne voyais pas comment rentrer à Melilla. Tout devient obscur d'un coup, tu peux pas réfléchir sereinement $\gg$.

La nouvelle d'un décès incite bien souvent les compagnons d'infortune du mort à lui « témoigner [leur] respect », comme me l'a expliqué un jeune Guinéen ayant participé à plusieurs commémorations. Un moment de recueillement collectif a été improvisé en l'honneur de Souleymane peu après le retour des délégués au campement. Au milieu des «bunkers », les membres de la communauté et certaines personnes venues des campements voisins ont partagé quelques minutes de silence. Il en a été de même après la confirmation du décès du «petit » Idrissa. À chaque fois, les participants ont souhaité avant tout marquer un temps d'arrêt dans les activités quotidiennes pour rendre hommage au compagnon perdu.

À la même époque, chaque semaine, d'autres cérémonies, mais à vocation œcuménique, étaient organisées quelque part non loin des campements par le prêtre de la Délégation des migrations. Des hommes et des femmes migrantes de différentes communautés se réunissaient pour exprimer leur respect aux défunts dans une communion spirituelle : «D'abord, quelqu'un prononçait une prière en accord avec la confession musulmane ; ensuite, je disais quelques mots et puis on gardait quelques minutes de silence pour les tombés [caídos] $\gg^{52}$. Parfois, quand les personnes présentes à la cérémonie les avaient connus, les morts étaient mentionnés par leurs noms, d'autres fois, ils étaient évoqués de façon générique comme tous les morts et les disparus de la traversée. Il s'agissait toujours de garder d'eux une trace à travers le récit et la prière. Pour Aristide Sessègnon, Béninois de 27 ans ayant participé à ces cérémonies, ces moments de communion permettaient de «manifester ensemble notre chagrin parce que c'est dur de voir des morts, des morts, beaucoup trop de camarades qui décèdent $\gg^{53}$. La dimension religieuse était importante pour lui, les prières contribuant à « soulager» la douleur. Pour d'autres, c'était avant tout la volonté de «ne pas oublier les disparus » qui les conduisait à se recueillir.

Lorsqu' Ibrahim Sagara (24 ans), originaire du Mali, a appris le décès de deux personnes à bord d'une petite embarcation partie de Tanger avec six passagers, il a été particulièrement touché. Il était censé partir avec eux, il avait même contribué à l'achat du canot gonflable, mais à la dernière minute il s'est désisté : "J'ai été sauvé par un instinct, une sensation. J'ai pas senti de monter dans le zodiac ». D'après ce qu'Ibrahim a appris par les survivants, les voyageurs avaient passé trois jours en mer, perdus et sans pouvoir faire fonctionner leurs téléphones. Ils avaient été secourus par les autorités marocaines et «le bateau [était] retourné au point de départ, mais retour avec deux personnes en moins ». Ibrahim Sagara ne connaissait que de vue

\footnotetext{
${ }^{50}$ Entretien avec Gaoussou Dembélé.

${ }^{51}$ Ibid.

${ }^{52}$ Entretien avec l'ancien responsable de la Délégation des migrations à Nador, Málaga, avril 2016.

${ }^{53}$ Conversation avec Aristide Sessègnon, Melilla, le 2 mai 2016.
} 
les deux hommes décédés, mais il a senti l'obligation de « faire quelque chose » pour eux : «Je me sentais mal mal, j'étais angoissé. Les frères étaient décédés, ça aurait pu être moi. Avec quelques combis [amis] on a pris un moment ensemble pour penser à eux, j'ai chanté quelques morceaux [Ibrahim est musicien] pour leur rendre hommage, témoigner notre solidarité. (...) Chacun a dit quelques mots sur les frères, ce qu'on savait d'eux, et puis la musique a fait du bien à tous ceux qui étaient là » ${ }^{54}$.

Représentation d'une absence, la cérémonie imprime une trace des défunts qui sera gardée par chacun des participants. Quoique plus intimes et éloignées de la vie publique, ces façons d'honorer la mémoire des morts font écho aux différentes manifestations organisées par des collectifs de soutien aux personnes migrantes un peu partout en Europe pour rendre hommage aux défunts tout en dénonçant le caractère mortifère des frontières. Ces « formes de persistance ou de résistance qui conduisent à pleurer les sans deuil » font ainsi irruption « à la fois pour contester les modèles à travers lesquels ces vies ne cessent de se voir dévaluées et pour affirmer leur valeur collective ${ }^{55}$. Largement partagées dans les réseaux sociaux et commentées dans certains médias, elles peuvent être également pensées comme une forme d' " activisme de la douleur $»^{56}$ en ce qu'elles transforment la souffrance en demande publique. Celle-ci est éminemment politique puisqu'elle cherche à porter un regard différent de celui véhiculé par les médias lorsqu'ils associent ces morts à des faits divers tragiques, voire à la représentation de la migration comme un fléau ${ }^{57}$. Dans ces récits alternatifs ou counter-narratives ${ }^{58}$, les morts sont érigés en symboles du régime contemporain des frontières ${ }^{59}$. Ils apparaissent comme une trace entendue comme « simulacre de présence » qui perturbe et disloque ${ }^{60}$ le discours hégémonique sur ces décès.

Annoncer le décès à la famille du défunt est une autre manière de lui rendre hommage. Tous mes interlocuteurs sans exception estiment qu'il s'agit d'un acte essentiel. Après l'identification du corps de Souleymane, plusieurs personnes du campement ont fait circuler la nouvelle de sa mort via Facebook. C'est ainsi qu'elles ont retrouvé le numéro de téléphone de son père à Bamako. Gaoussou Dembélé l'a appelé et la famille a appris le sort de ce jeune parti vers l'Europe plus d'un an auparavant : "C'était un moment très très dur, mais il fallait le faire ». C'est également par Facebook que les survivants de l'embarcation perdue au large de Tanger ont pu contacter un ami de l'un des morts qui a contacté la famille de ce dernier. Aucune trace n'a pu être retrouvée de l'autre jeune décédé au cours de cette traversée vers les côtes andalouses : «Personne n'avait des informations, son nom, son village. Il était personne », regrette Ibrahim Sagara. Avertir les proches est une façon de contrer l'effacement total auquel beaucoup de mes interlocuteurs ont avoué vouloir échapper à travers leur migration ${ }^{61}:$ « $\mathrm{Si}$

\footnotetext{
${ }^{54}$ Conversation avec Ibrahim Sagara, Alméria, le 12 mai 2016.

${ }^{55}$ Judith Butler, Qu'est-ce qu'une vie bonne?, Paris, Payot, 2014, p. 64.

${ }^{56}$ Maurice Stierl, « Contestations in Death: The Role of Grief in Migration Struggles », Citizenship Studies, 20 (2), 2016, p. 173-191.

${ }^{57}$ Sur ces représentations médiatiques, voir Lynda Mannik (ed.), Migration by Boat. Discourses of Trauma, Exclusion, and Survival, New York, Berghahn Books, 2016.

${ }^{58}$ Leanne Weber, Sharon Pickering, Globalization and Borders: Death at the Global Frontier, Basingstoke, Palgrave Macmillan, 2011, p. 70.

${ }^{59}$ L'image du corps inerte du petit Alan Kurdi échoué sur une plage turque est devenue, pour reprendre les distinctions de la sémiotique de Pierce, une icône représentant les effets des politiques européennes en matière d'immigration. Charles Pierce, Écrits sur le signe, Paris, Le Seuil, 1978.

${ }^{60}$ Jacques Derrida, « La différance », dans J. Derrida, Marges de la philosophie, Paris, Éditions de Minuit, 1972 , p. 25.

61 Sur ce sentiment d'inexistence comme moteur de la migration, voir, parmi d'autres, Mahamet Timera, «Aventuriers ou orphelins de la migration internationale ? Nouveaux et anciens migrants "subsahariens" au Maroc », Politique africaine, 115, 2009, p. 175-195; M. Timera, «Les migrations des jeunes Sahéliens: affirmation de soi et émancipation », Autrepart, 18 (2), 2001, p. 37-49 ; Knut Graw, « On the Cause of Migration: Being and Nothingness in the African-European Border Zone », dans Knut Graw, Samuli Shielke (eds), Global
} 
l'information reste à la frontière ce n'est pas bon. C'est pas bon pour la famille qui ne sait rien, qui attend toujours des nouvelles. Et c'est pas bon pour le mort, il est oublié peut-être, il finit oublié. C'est pas bon. Nous voulons tous que notre famille sache si on est mort. (...) Gaoussou a parlé, nous étions à côté de lui. C'était dur mais ça nous a fait du bien, son âme pouvait reposer $»^{62}$.

Contacter la famille, c'est contribuer à ce qu'elle puisse traiter le défunt. Plus encore que laisser une trace, annoncer le décès est une façon de restituer au mort une certaine traçabilité. Même si elle ne connaît pas l'emplacement final de la dépouille, la famille sait que le proche est décédé et que ce décès est survenu dans un lieu et à un moment précis. Son itinéraire et son histoire peuvent alors être retracés, du moins en partie. Et lorsque le corps n'est pas rapatrié, comme dans le cas de Souleymane, le mort est en quelque sorte « reterritorialisé » ${ }^{63}$ puisqu'il peut être réintégré parmi les siens : "Aujourd'hui, en Afrique de l'Ouest, il y a beaucoup de mères qui sont assises pendant quinze ans qui disent : "Mon enfant est parti à l'aventure pour chercher de l'argent, pour nous l'envoyer". Qui ne savent rien du sort de leur enfant, vivant ou mort elles savent pas ${ }^{64}$.

Quand elle apprend le décès d'un proche, la famille peut lui attribuer la place et le statut particuliers qu'acquièrent les morts au sein de sa société ${ }^{65}$, contrairement aux disparus qui n'existent que comme énigme. Des stratégies simples sont mises en place pendant la traversée qui font office de remède à l'oubli absolu. Par exemple, apprendre par cœur le numéro de téléphone ou le nom d'un proche d'un compagnon de voyage afin de prévenir la famille s'il le faut. Naba Tigré (23 ans), Burkinabé d'origine bissa, l'exprimait dans ces termes : « Beaucoup trop de frères meurent sur la route vers l'Europe, beaucoup disparaissent dans la mer, beaucoup trop disparaissent à la barrière, beaucoup périssent dans le désert; je ne veux pas disparaître sans laisser de trace. (...) Je ne veux pas mourir, mais si je meurs j'aurais tenté et je veux que ma famille l'apprenne, qu'ils sachent que j'ai tout donné pour avancer dans la vie. J'ai dit à mon frère [il fait référence à un jeune Bissa comme lui, originaire d'un village proche du sien, compagnon de route depuis plus d'un an qui connaît le numéro de téléphone de sa mère] que s'il m'arrive quelque chose qu'il appelle, qu'il dise que j'ai fait tout ce que j'ai pu pour ne pas disparaître complètement ${ }^{66}$.

La trame narrative développée dans ces pages a été construite autour de la mort de Souleymane et de son traitement. En filigrane, la notion de trace a été mobilisée comme un outil permettant de saisir ensemble différentes dimensions de la prise en charge des morts aux frontières. De la gestion des corps retrouvés au traitement des corps absents, en passant par les modalités d'honorer les personnes décédées, il s'agit à chaque fois de retrouver, de suivre ou de laisser des traces plus ou moins palpables, plus ou moins visibles. Dans cette palette de perceptibilité se rejoignent la trace comme indice permettant l'identification des corps retrouvés et la trace

Horizon: Expectations of Migration in Africa and the Middle East, Louvain-La-Neuve, Leuven University Press, 2012, p. 23-42.

${ }^{62}$ Conversation avec Amadou Coulibaly.

${ }^{63}$ Sur la territorialisation des morts et plus particulièrement sur leur « déterritorialisation », voir Grégory Delaplace, L'invention des morts. Sépultures, fantômes et photographies en Mongolie contemporaine, Paris, Centre d'études mongoles et sibériennes-École pratique des hautes études, 2009.

${ }^{64}$ Entretien avec Amadou Coulibaly.

${ }^{65}$ Maurice Bloch, Jonathan Parry, Death and the Regeneration of Life, Cambridge, Cambridge University Press, 1982.

${ }^{66}$ Conversation avec Naba Tigré, Melilla, le 17 avril 2016. 
comme représentation d'une absence qui se manifeste à travers les commémorations, les témoignages qui cherchent à contrer l'oubli, ainsi que les récits qui rapportent le décès aux proches du défunt, contribuant ainsi à reterritorialiser sa mort ${ }^{67}$. Si ces traces sont intéressantes à ethnographier, c'est parce qu'elles condensent des histoires et des régimes esthétiques ${ }^{68}$ qui permettent de saisir la politique en matière de migrations telle qu'elle se déploie et est vécue par celles et ceux qui traversent et qui habitent les frontières européennes ${ }^{69}$.

Le récit de la mort de Souleymane et de son traitement a été construit à partir de plusieurs voix et fragments de discours décousus, recueillis notamment auprès de quelques-uns de ses compagnons. Si ces personnes m'ont aussi facilement raconté leurs histoires, c'est sans doute parce que la mort est omniprésente pendant la traversée vers l'Europe. C'est peut-être aussi parce qu'elles ont saisi l'anthropologie comme un moyen leur permettant d'imprimer une trace de leurs expériences de vie et de mort à la frontière.

\footnotetext{
${ }^{67}$ On y trouve également des traces qui échappent en partie à l'anthropologue, les traces mnésiques dérobées qui s'inscrivent pourtant chez celles et ceux qui ont assisté, de près ou de loin, à la mort de pairs ainsi que les traces des corps qui s'effacent lorsqu'ils disparaissent, telles des laisses de mer ou «tidemarks », pour emprunter le terme à S. Green dans « Lines, Traces and Tidemarks: Reflections on Forms of Borderlines », cité.

${ }^{68}$ J'utilise ici la notion d'esthétique telle que proposée par Jacques Rancière, comme « un découpage des temps et des espaces, du visible et de l'invisible, de la parole et du bruit qui définit à la fois le lieu et l'enjeu de la politique comme forme d'expérience ». Jacques Rancière, Le partage du sensible. Esthétique et politique, Paris, La Fabrique, 2000.

${ }^{69} \mathrm{~J}$ 'emprunte ici l'idée de traces comme formes de condensation à V. Napolitano, « Anthropology and Traces », art. cité.
} 